\title{
The SSC Full Cell Prototype String Test
}

\author{
P. Kraushaar, W. Burgett, L. Cromer, J. Gannon, D. Haenni, \\ M. Hentges, T. Jaffery, M. Levin, A. McInturff, G. Mulholland, D. Revell, \\ D. Richter, W. Robinson, D. Voy, J. Weisend II, and J. Zatopek \\ Superconducting Super Collider Laboratory ${ }^{*}$ \\ 2550 Beckleymeade Ave. \\ Dallas, TX 75237, USA
}

November 1994

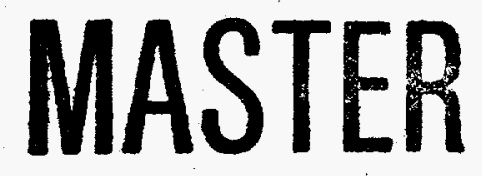

*Operated by the Universities Research Association, Inc., for the U.S. Department of Energy under Contract No. DE-AC35-89ER40486. 


\section{DISCLAIMER}

This report was prepared as an account of work sponsored by an agency of the United States Government. Neither the United States Government nor any agency thereof, nor any of their employees, make any warranty, express or implied, or assumes any legal liability or responsibility for the accuracy, completeness, or usefulness of any information, apparatus, product, or process disclosed, or represents that its use would not infringe privately owned rights. Reference herein to any specific commercial product, process, or service by trade name, trademark, manufacturer, or otherwise does not necessarily constitute or imply its endorsement, recommendation, or favoring by the United States Government or any agency thereof. The views and opinions of authors expressed herein do not necessarily state or reflect those of the United States Government or any agency thereof. 


\section{DISCLAIMER}

Portions of this document may be illegible in electronic image products. Images are produced from the best available original document. 


\title{
The SSC Full Cell Prototype String Test
}

\author{
P. Kraushaar, W. Burgett, L. Cromer, J. Gannon, D. Haenni, M. Hentges, T. Jaffery, M. Levin, A. D. McInturff $†$, G. \\ Mulholland, D. Revell, D. Richter, W. Robinson, D. Voył, J. Weisend II, and J. Zatopek \\ Superconducting Super Collider Laboratory*, Dallas, Texas 75237
}

\begin{abstract}
At the conclusion of the SSC half cell magnet string testing program in February, 1993, the prellminary data analysis revealed that several substantive technical questions remained unresolved. These questions were: 1 ) could the high voltages to ground ( $>2 \mathrm{kV}$ ) measured during fault (quench) conditions be substantially reduced, 2) could the number of magnetic elements that became resistive (quenched) be controlled and 3) did the cryostats of the magnetic elements provide adequate insulation and isolation to meet designed refrigeration loads. To address these and other existing questions, a prototypical full cell of collider magnets (ten dipoles and two quadrupoles) was assembled and tested. At the conclusion of this testing there were definitive answers to most of the questions with numerical substantiation, the notable exception being the heat leak question. These answers and other results and issues are presented in this paper.
\end{abstract}

\section{INTRODUCTION}

The Superconducting Super Collider Laboratory (SSCL) has conducted a magnet string testing program aimed at verifying the performance requirements for the superconducting components and the supporting systems necessary for their operation and control in an accelerator environment. During this program, operational experience was gained with the magnets and the supporting systems. The initial string tests using $\mathbf{4 0} \mathrm{mm}$ aperture magnets were conducted at Fermi National Accelerator Laboratory (FNAL). The "ER" string was located at the E-4 service building site of the Tevatron accelerator and has been previously described [1],[2]. The second magnet string to be tested was the half cell of $50 \mathrm{~mm}$ aperture SSC prototypes assembled at the $\mathrm{N}-15$ site, near Waxahachie, in Ellis County, TX. The Accelerator Systems String Test (ASST) was initially assembled to meet a Congressionally mandated milestone to demonstrate the operation of a prototypical collider half cell at design parameters. The ASST achieved the designated milestone on August 14, 1992. The results from this demonstration (referred to as Run 1) have been previously presented [3],[4]. After the completion of Run 1, the half cell string was warmed for some minor electrical, valve, pipe routing and insulation modifications but no magnet configuration changes. Near the end of October, 1992 an extensive testing program (Run 2) on the half cell string was initiated. The program lasted until February, 1993

\footnotetext{
* Operated by the Universities Research Association, Inc. for the U.S. Department of Energy under contract number DE-AC35-89ER40486. †Present Address: Lawrence Berkeley Laboratory $\neq$ Present Address: Fermi National Accelerator Laboratory

Manuscript received October 16, 1994.
}

when operations were suspended to begin the reconfiguration of the string into a full collider cell. The results from this testing period have been described earlier [5],[6].

At the conclusion of the half cell testing program, the data analysis indicated that at least four substantive questions remained unresolved. These were 1) could the high voltages to ground $(>2 \mathrm{kV})$ measured during fault or quench conditions be reduced by matching the magnet residual resistivity ratio (RRR) within the quench protection units (quarter cells), 2) could the fault (quench) condition be contained within a protection unit, 3) were the heat leak rates to the cryogenic system from the cryostats containing the magnetic elements within design values, and 4) was the magnetic field margin (percentage of critical value) of the magnetic elements adequate at high currents? To address these questions, as well as other operational issues, a prototypical full cell of collider elements was assembled into a $188 \mathrm{~m}$ unit. The cell consisted of ten dipoles, two quadrupoles, a mid cell (SPR) spool containing magnetic correctors, cryogenic control valves, a vacuum isolation barrier, safety valves, quench bypass leads and $R \& D$ sensors, and two end spools for power and cryogenic feeds. Operational experience and test data were collected from the full cell during two periods. The first segment of Run 3 testing began at the end of August, 1993 and lasted through October, 1993 when operations were suspended due to the SSC project cancellation. This testing period was devoted to string checkout and thermal related measurements. Since the critical power testing phase of the full cell test program had not started, a proposal for a six week power testing period was submitted to the Department of Energy (DOE) for funding approval under the termination guidelines. The proposal was approved by DOE in Washington and the final testing period for Run 3 began on January 10, 1994.

At the conclusion of testing, definitive answers to three of the four questions had been obtained with adequate numerical support. The exception was the cryostat static heat leak rate values. The rates measured were consistent with the previous half cell results, but did not provide a clear answer due to the presence of very high spool piece heat loads. The matching of magnets within a quench protection unit with similar low temperature normal state resistances (RRR) of their outer coils, reduced peak voltages to ground by approximately 400 volts. The operational parameters required to contain the quench to within a protection unit are understood, demonstrated and will be discussed. Some data were taken to establish if the quench protection unit could be enlarged to a half cell. The magnetic field margin is clearly satisfactory with the cell having operated uneventfully to 


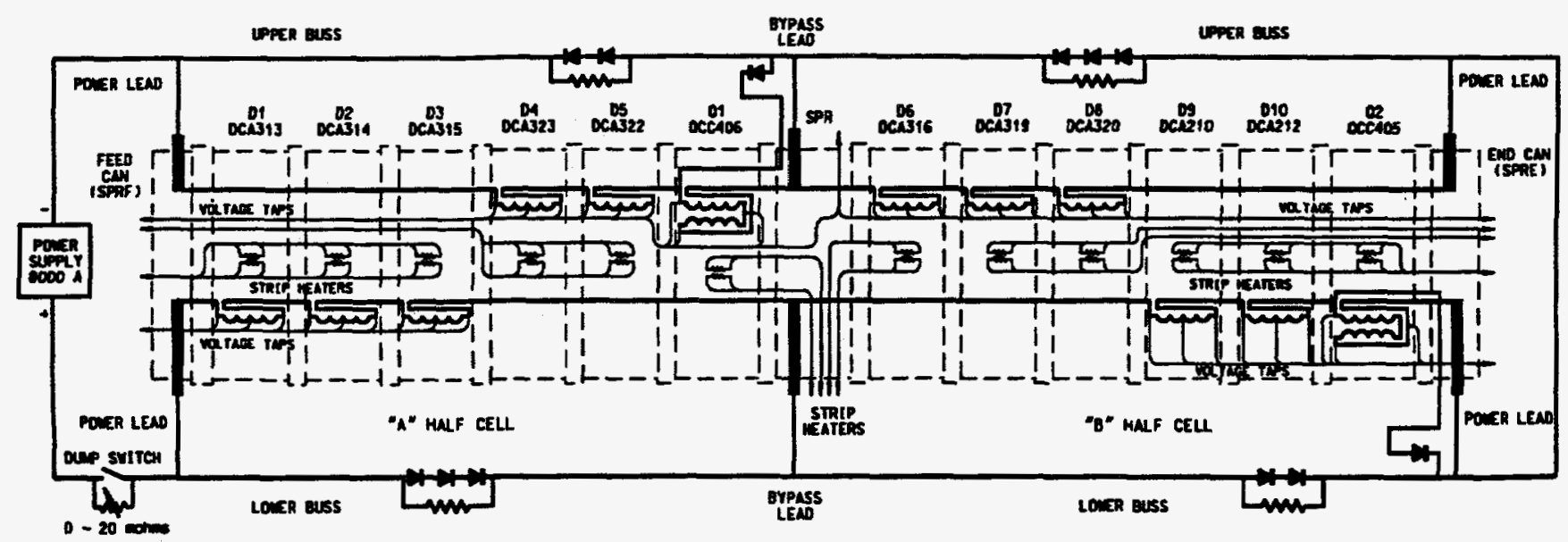

Fig 1. Generalized electrical schematic of the ASST full cell showing order and placement of components and principle quench protection elements. For quench protection purposes, the circuit was logically composed of four quarter cells composed of D1, D2, D3; D4, D5, Q1; D6, D7, D8; and D9, D10, Q2. A quench detected in any one quaner cell fired all the protection heaters in that unit. The dump switch was an SCR backed up by a mechanical switch in series.

greater than 7000 amperes. The test results will be discussed in detail with an emphasis placed on making a clear division between experimentally determined (measured) numbers, and the various interpretations derived from them.

\section{EXPERIMENTAL PROCEDURE}

The full cell string components were installed and connected electrically as shown in Fig. 1 without regard to the direction of the magnetic field. The only configuration consideration was that magnets with similar low temperature normal state resistance in the outer coils were grouped into protection units. Table I contains the RRR values for the dipole outer coils used in the full cell, grouped by protection unit. The SS values are from short sample tests on the cable. The "Test" values were measured on the completed magnets. Electrically, the full cell was divided into quarter cell protection units which were composed of three dipoles or two dipoles and a quadrupole. Due to the various integrated energy density capacities of the cables used to fabricate the magnet coils, $\left(20,16\right.$, and $10 \times 10^{6} \mathrm{~A}^{2}$-s for the dipole inner, outer and quadrupole coil cables, respectively), the quadrupoles required an individual bypass lead and diode configuration. As each element was installed, the various electrical circuits were checked for correct component placement and high voltage integrity. After the successful completion of these preliminary checkouts, the cryogenic connections in the interconnect region were welded and an initial leak check done. Upon completion of installation of each half cell, the cryostat insulating vacuum enclosure was closed and pumped down. The cold mass was pressurized and leak checked with respect to the insulating vacuum. The interconnects were then reopened, insulated, and closed. Upon completion of the full cell assembly, another $0.92 \mathrm{MPa}$ pressurized vacuum leak test was done. After the system passed this test, the cold mass, liquid He return, and $20 \mathrm{~K} \mathrm{He}$ shield lines were pressurized to $1.85 \mathrm{MPa}$ for high pressure certification while the insulating vacuum was still connected to the leak detectors. The highest quench pressure observed during testing in the magnet cold mass transducers was 1.18 MPa. Lower pressures were observed in the other cryogenic circuits. Following the successful completion of leak and pressure qualification tests, the string was cooled to liquid helium temperature, and a cold verification of the electrical circuits completed. An electrical integrity failure was detected during this check. This problem was electrically isolated to the SPR spool. Photographs taken during the spool manufacturing process provided a consistent and probable physical location. A removal procedure was developed and later successfully completed allowing the string to be qualified for power testing [7].

During the thermal testing period, the cell was subjected to a series of operational performance characterizations and heat load measurements. The string cool down was accomplished in 6 days and considered thermally stable enough in 3 weeks to perform the static heat leak measurements. During this stabilization period, the electrical check out of the string systems was completed. In addition,

TABLE I

MEASURED RRR VALUES FOR THE FULI, CELLDIFOLE MAGNETS

\begin{tabular}{|c|c|c|c|c|}
\hline Magnet & \multicolumn{2}{|c|}{ Upper Outer } & \multicolumn{2}{|c|}{ Lower Outer } \\
\hline & SS & Test & SS & Test \\
\hline DCA313 & 39 & 174 & 38 & 171 \\
\hline DCA314 & 41 & 177 & 38 & 174 \\
\hline DCA315 & 41 & 173 & 41 & 174 \\
\hline DCA323 & 39 & 102 & 39 & 121 \\
\hline DCA322 & 40 & 113 & 40 & 112 \\
\hline DCA316 & 39 & 109 & 39 & 109 \\
\hline DCA319 & 42 & 96 & 42 & 97 \\
\hline DCA320 & 39 & 98 & 39 & 99 \\
\hline DCA210 & 41 & 217 & 41 & 209 \\
\hline DCA212 & 37 & 231 & 37 & 227 \\
\hline
\end{tabular}


spool piece recooler was extensively tested for control and performance parameters. For thermal testing, the operational cyrogenic environment was super critical $\mathrm{He}$ gas at $4.5 \mathrm{~K}$ at $405.2 \mathrm{kPa}$. The mass flow rate was varied between $20 \mathrm{~g} / \mathrm{s}$ and $50 \mathrm{~g} / \mathrm{s}$ depending on the measurement.

The feed and end spools had been further modified during the half to full cell reconfiguration period to reduce their thermal loads to the magnet string. This was an attempt to improve their operational performance. A new SPR spool was used (similar in design to the one used in the half cell tests) which had not been fully tested at LHe temperatures prior to installation due to schedule constraints. The spool pieces, particularly the SPR, still represented the dominant heat load into the cold mass He circuit, resulting in a loss of accuracy in the static heat load determinations for the individual low loss elements. After the completion of thermal testing, while waiting for permission to proceed to the full cell power tests, the string was allowed to warm up to $80 \mathrm{~K}$, with only the $\mathrm{LN}_{2}$ system maintaining the string.

The full cell power commissioning procedures used to reach the maximum operating current, and beyond, were the same as previously reported [4],[5] except there were two half cells to check. The cryogenic environment was similar to the thermal testing one except that the He mass flow was a nominal $100 \mathrm{~g} / \mathrm{s}$, and the temperature varied between $3.8 \mathrm{~K}$ and $4.6 \mathrm{~K}$. In the process of qualifying the string, the data collected and operations experience gained, answered the key questions on reduction of voltages to ground and quench containment. Table II presents the number of events, sorted by type, for which data were obtained, catalogued, and stored from the ASST fast circular buffer data acquisition systems.

A series of seven protection (strip) heater induced quenches was conducted to study the quench response of the magnet to three different strip heater designs. The heater design parameters were the same except for the pad length. Each strip heater contains twelve heater pads, and each heater circuit contains two strips. The standard heater had a $610 \mathrm{~mm}$ long pad while the two experimental low energy designs had pad lengths of $50 \mathrm{~mm}$ and $100 \mathrm{~mm}$. Two dipoles (DCA322 and DCA 323) each contained one standard heater circuit and one of the experimental designs, respectively. The low energy design reduced, up to an order of magnitude, the energy required in the heaters to reach a given temperature (same pad time integrated energy density). Quenches were induced in each of the two magnets using both standard and low energy heaters at low $(3000 \mathrm{~A})$ and high $(6000 \mathrm{~A})$ magnet cell currents. The energy delivered to the heater circuits in each magnet in the full cell had previously been adjusted to give approximately the same thermal diffusion time or delay from heater firing until resistance appeared in the outer coil windings. The energy was adjusted to produce coil resistance in less than $250 \mathrm{~ms}$ at $2000 \mathrm{~A}$, with typical values being around $150 \mathrm{~ms}$ to $200 \mathrm{~ms}$.

A series of spot heater induced quenches was conducted, as well, to simulate spontaneous quench conditions at various magnetic fields. These types of resistive transitions typically lead to the highest MIIT values that have been observed [8]. The initial spot heater quenches were used to verify that at least the minimum energy required to initate a quench was being delivered to the spot heaters. During these tests, a delayed protective measure was employed where the quench protection system (QPS) automatically fired the protection heaters a hundred milliseconds or so after the spot heaters were fired. This delayed operation was disabled once it was verified the spot heaters would induce a quench. The strip heaters were then activated only after the QPS detected a resistive transition in one of the half coils. This was the normal operational sequence for the QPS [9]. This series concluded with a spot heater induced quench in D10 with the string powered to $7 \mathrm{kA}$. This was the highest magnetic field achieved by the full cell and corresponded to approximately 7 tesla. This current level exceeded the field required for $20 \mathrm{TeV}$ operations [10].

Besides the spot and individual quench heater tests, a sequence of special quenches was conducted. These included quenches initiated by firing all the quench heaters simultaneously in up to a half cell. Also, two quenches were done with a modified bypass circuit configuration in which all upper buss magnets were in a single quench protection unit. This configuration was achieved by disconnecting the bypass lead for the upper buss at the SPR. The logical response of the QPS was modified to accommodate this circuit change. The logic was tested first at a low current to insure the safety of the string.

The last experiment performed during Run 3 was to degrade the insulation vacuum in the second half cell in a controlled fashion to mimic a catastrophic failure. The insulating vacuum between the two half cells was isolated by the vacuum barrier in the SPR spool. Air was admitted to the insulating vacuum by opening an electrical gate valve (25.4 $\mathrm{mm}$ aperture) located at the interconnect between D6 and D7 in the second half cell. The gate valve aperture was restricted by an orifice consisting of a $15 \mathrm{~cm}$ long, $9.5 \mathrm{~mm}$ ID tube. The string was isolated from the refrigerator prior to the test for safety reasons. The valve was opened for approximately fifty-four (54) minutes. Data were acquired on the three data acquisition systems at the ASST at rates ranging from 3.3 $\mathrm{mHz}$ to several hundred hertz during this period. The standard data logging system continued to record data a 5

TABLE II

RUN 3 TEST EVENTS SORTED BY TYPE

\begin{tabular}{ccc}
\hline Quench Events & Spontaneous & 56 \\
(DCA 212 @ 6.00 kA \& DCA 313 @ 6.36 kA) & 2 & \\
Strip Heater Induced & 42 & \\
Spot Heater Induced & 12 & \\
Mechanical Swritch Failures (Induced Down Ramps) & & 10 \\
Scheduled Down Ramps for Calibrations & 2 \\
Vacaum Degradation Test & 1 \\
Total Data Events & 69 \\
\hline
\end{tabular}


minute intervals during the warm up process. After the test, the insulating vacuum was restored with vacuum pumps.

\section{RESULTS}

The measured maximum voltages to ground were reduced by matching the RRR of the outer coils of each magnet within each protection unit (quarter cell). These results are shown in Fig. 2 and compared to those from Runs 1\&2. In the full cell, the RRR values were matched to approximately $10 \%$ of the maximum outer coil RRR in the protection unit, whereas in the half cell tests, the outer coil RRR varied by approximately a factor of 2 . Other options are available which may further reduce these voltages. For example, better techniques (electronic or numeric) for the detection of the onset of the resistive transition would decrease the quench detection threshold without increasing nonevent alarms. Note that, due to an electronic problem, the quench detection threshold for matched RRR data was $1.0 \mathrm{~V}$, whereas it was $0.5 \mathrm{~V}$ for the unmatched RRR data. Thus the voltage to ground reduction from matching the low temperature normal state resistance may be greater than presented.

Once the mechanism for quench propagation in the full cell was understood, quench containment became straightforward. Secondary quenches occurred when the current down ramp rate exceeded the minimum rate at which an ASST magnet would quench [11]. This results if a significant percentage of the inductance in the power circuit is removed as when a quenching section is bypassed. For the full cell circuit this meant that the dump resistor value had to be appropriately reduced as string currents increased. This mechanism became apparent during a string commissioning strip heater induced quench in D6. D6 quenched as expected, but $\mathrm{D} 3$ also quenched during the down ramp which followed. The quarter cell containing D3 was separated by a quarter cell of nonquenching magnets from the D6 cell. It was cryogenically upstream and thus colder. The magnets in between, although warmer, had exhibited less sensitive ramp rate behavior in single magnet quench tests [12],[13]. The dump resistor value was adjusted and in a subsequent quench, with a down ramp rate of 265 amperes/second, the induced quench was confined to the third quarter cell where it was initiated. Therefore the causal question of quench growth from protection unit to unit had been answered.

The maximum MIITS value was observed in D10 (DCA212) during a spot heater induced quench at 7035 amperes, resulting in 13.5 MIITS being absorbed. This corresponds to an adiabatic temperature of $430 \mathrm{~K}$ with the actual probably $10 \%$ less [16]. These values are well within the coil conductor specification. The MIITS data calculated from measured current profiles for strip heater induced quenches are given in Fig. 3.

The statistical significance of the heat load data obtained from the full cell was no better than that obtained in the half cell runs. This was due to the high heat loads present in the

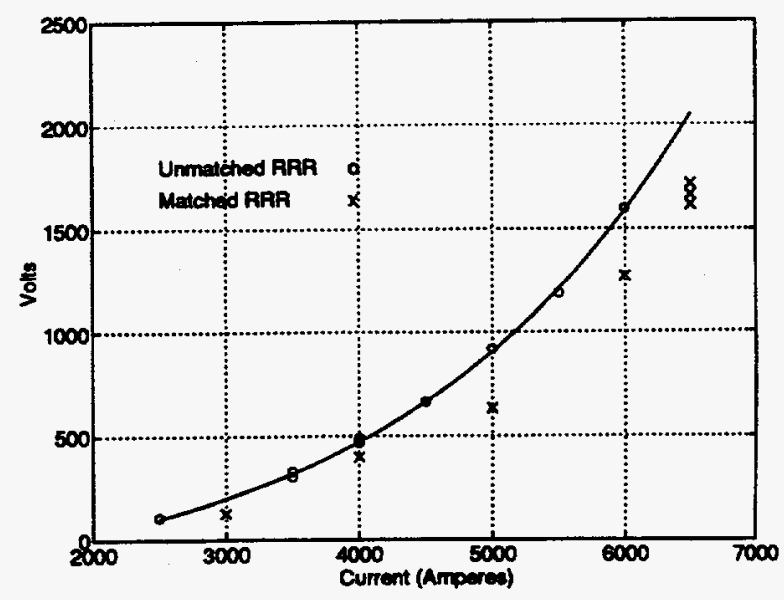

Fig. 2. Comparison of the measured maximum voltage to ground observed in Runs 1 \&2 (unmatched RRR, variation $\sim 2$ ) versus those measured in Run 3 (matched RRR, variation 10\%).

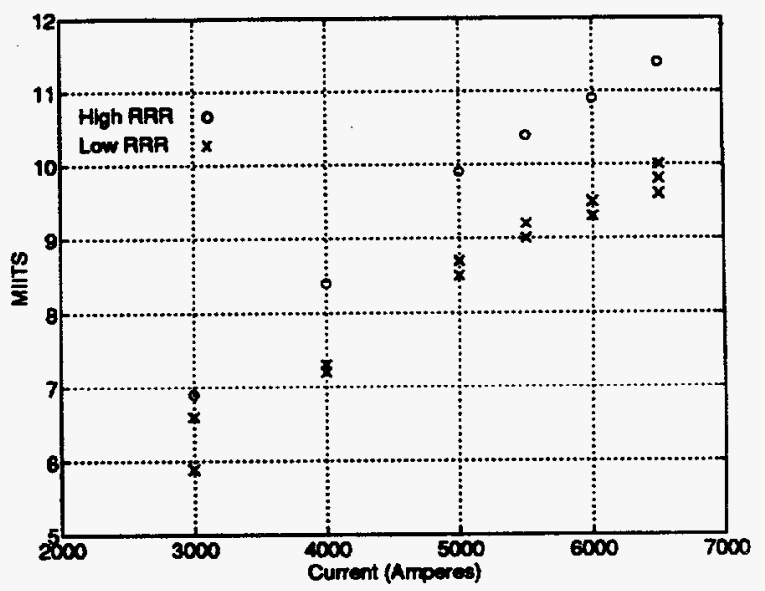

Fig. 3. MIITS values calculated from the measured current profiles versus maximum string current for strip heater induced events showing that high RRR magnets experience higher MIITS than low RRR magnets due to the resulting longer current decay times.

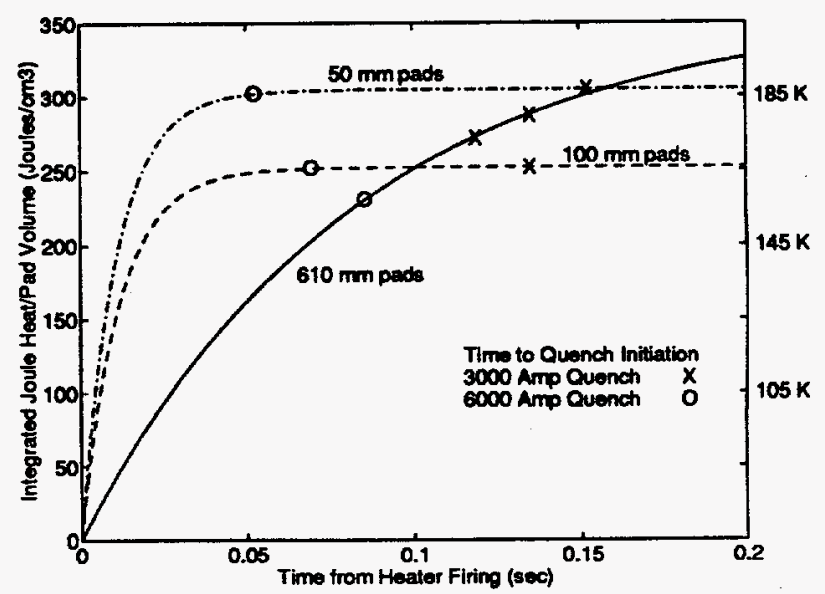

Fig. 4. Integrated Joule heating energy per unit heater pad volume giving the total energy density in the strip heaters from the time of heater firing as a function of time. 
cold mass and shields circuits of the SPR. The measurement accuracy was similar to the half cell tests and produced essentially the same results [6]. To summarize, the heat load into the three middle dipoles of each half cell averaged $1.25 \pm .5$ watts per dipole when the $20 \mathrm{~K}$ shield system was operating around $20 \mathrm{~K}$. The design value was 0.36 watts. The 20K shield loads were closer to the design requirement of 5.06 watts. The " $A$ " and " $B$ " half cell loads were $4.93 \pm 0.4$ watts and $6.13 \pm 0.7$ watts per dipole, respectively. The observed quadrupole heat load to the $20 \mathrm{~K}$ shields of 6.8 watts and 13.8 watts for $Q 1$ and $Q 2$, respectively, was higher than the design value of 2.68 watts, but both were connected to high loss spools. This may indicate a possible problem. No data was obtained on the $80 \mathrm{~K}$ shield system during Run 3 due to a lack of stability in the new string $\mathrm{LN}_{2}$ system. The heat load measurement technique was checked by using internal calibration heaters. The measured values agreed to within $4 \%$ of the calculated input heat and compared well to the previous run's values of $4 \%$ to $6 \%$.

The remaining major question asked in this run was whether the operating field margin of the magnets, the dipoles in particular, was adequate. The full cell was powered to above 6900 amps twice. The first time, a power supply over-current trip activated the dump circuit and ramped the string down. The second time the string was taken to a current of 7035 amperes and a spot heater quench in D10 was induced. This quench was contained in the fourth quarter cell. The peak temperature in the magnet string prior to quench initiation was $0.24 \mathrm{~K}$ lower than the magnet design operating temperature of $4.35 \mathrm{~K}$. The two spontaneous quenches that occurred were both at greater than $90 \%$ the peak operational current. One dipole had been involved in a maximum MIITS test just prior to installation in the ASST. The other dipole had run in the half cell testing without incident. Given the operational experience and test results, the peak magnetic field margin seems adequate with the cell not limited even at $7 \mathrm{~T}$.

The data from the strip heater study clearly demonstates the importance of the electrical circuit characteristics of the strip heaters in QPS design. The data show that the time required for the heat to develop in the high energy strip heater (standard design) to quench the windings at low magnetic field $(-3 T)$ was slightly less than the time for the low energy, experimental heaters. However, the relationship reversed by the time the magnetic field reached $6 \mathrm{~T}$. The cause of this reversal is clear when the integrated energy densities (adiabatic temperature) of the heaters are plotted versus time (Fig. 4). The time constants for the circuits with the experimental heaters permitted the critical energy densities to be reached sooner in the heater pads. This indicates that at higher currents, the thermal diffusion times between the heater and the windings of the magnet becomes the limiting factor to quench initiation.

The final Run 3 test was to raise the insulating vacuum pressure of the cryostat on one side of the vacuum break to

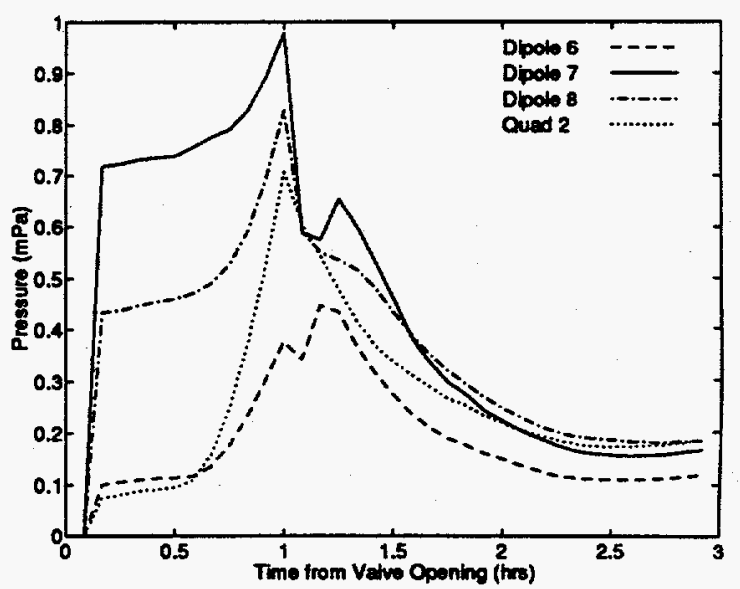

Fig. 5. The pressure response of the magnet cryostat insulating vacuum to the induced air leak as a function of time from event initiation.

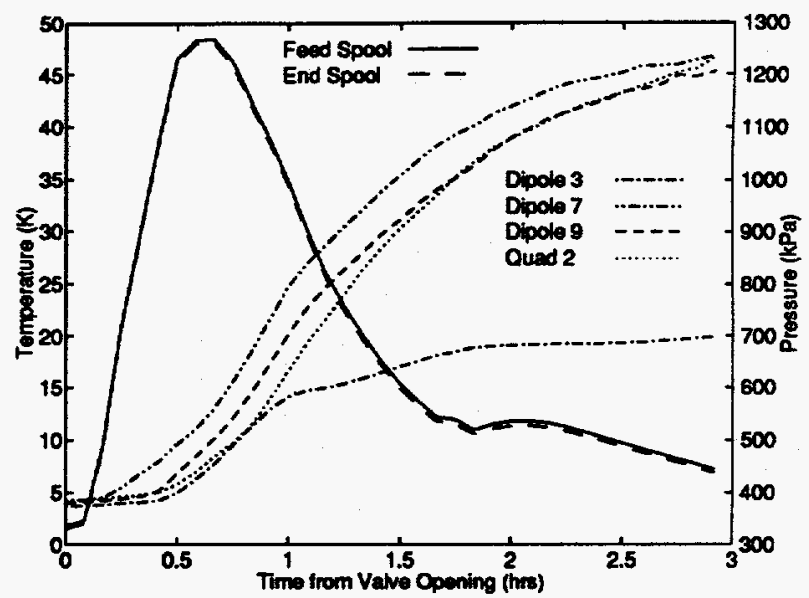

Fig. 6. The cold mass pressure measured at the feed and end spools, and the magnet cold mass temperature observed at four locations along the cell as a function of time from the leak initiation. The dipole 7 temperature sensor was the closest to the leak point. The insulating vacuum in the first half cell (dipole 3) remained intact.

simulate a failure of that system. This type of test has not been performed previously on actual systems because of the implied risk to the equipment. Both helium and air leaks can degrade the insulating vacuum. Since the ASST string was expendable, this test was performed to provide actual data on this type of failure for future design considerations. Air was chosen for this test since its impact was thought to be more severe. As the data shows, the system response was not catastrophic. The insulating vacuum response to the air leak as a function of time is given in Fig. 5, and the cold mass temperature and pressure response from the resulting heat influx are shown Fig. 6 . At the peak cold mass pressures, the He return line safety relief valve opened and vented cold gas. The valves cycled several times during the 54 minute test.

\section{DISCUSSION \& SUMMARY}

The impact of a variation in the RRR of the outer magnet coils on the voltages to ground observed during a quench has 
been established. The control of the manufacturing process and materials to maintain the uniformity and reproducibility of the superconducting cable and the coil winding production environment should result in a more uniform normal state resistance of the coils. This uniformity will certaintly result in lower voltages with respect to ground for quenching magnet strings. There are other factors which will affect the magnitude of these voltages. The design of the protection heaters is one which also has system consequences. The heater impedance can be adjusted to optimize energy density development versus discharge time, so that the quench initiation time is dominated by the thermal diffusion time constant of the electrical insulation between the heater and coil. Optimizing the heater volume distribution in the windings affects quench development as well. The system impact of lowering of the strip heater energy required to quench the coils is to reduce the physical size, and cost, as well as to increase the reliability of the QPS. Another factor to consider is the time it takes to detect resistive transitions. Experience from Run 3 suggests that future quench protection systems would be more effective if a field and ramp rate dependent reactive term for detecting coil resistance is included in the design.

The localization (containment) of a quench to a protection unit was primarily dependent on whether the minimum down ramp rate for quenching one of the remaining magnets had been exceeded. The minimum rate is influenced by two factors. The first is the coupling between the cable strands. A decrease in this coupling allows much higher quench threshold ramp rates [14],[15]. There are other reasons for decreasing the inter-strand coupling besides quench containment some of which are ramp rate losses and ramp dependent distortion of the field harmonics. The other factor is the total inductance of the ramping unit. Twenty-four cells were proposed per unit for the collider. Therefore, a quenching quarter cell would only change the inductance by about $1 \%$ compared to the $25 \%$ in the ASST cell test.

Clearly there has not been a resolution of the cryostat questions. The cryostat losses are high for the cold mass but manageable, and may be easily improved with a better shield mechanical design. Shield interferences were noted during string assembly and corrected in the interconnect regions. Correcting the spool heat leak problem will be more difficult because it appears to stem from poor design and manufacturing techniques.

The results of the insulation vacuum failure test at the termination of the power tests were very benign. The "SPR" vacuum isolation barrier functioned properly as did the pressure relief valves. The observations are clearly not in agreement with failure response models that were used for safety design calculations that predicted catastrophic results. This experiment should be repeated with helium which would not have frozen out in between the layers of the MLI blankets. This is presumably the cause for the observed self limiting behavior.

\section{ACKNOWLEDGMENT}

The authors express their appreciation and gratitude to the technical crew lead by $C$. White, who did an excellent job on the mechanical assembly of the full cell; the electrical engineering systems group lead by R. Abel, for the rapid and accurate reconfiguration of the quench protection system; the cryogenics operations crew for their around the clock support and ability to have the string ready for the next test before the experimenters were; and to all of our colleagues who continued to support this run under the very difficult conditions of a canceled project. Also the crucial effort and support, on our behalf, by interim SSCL Director, Dr. J. Peoples and DOE's Chief Scientist for the SSC, Dr. G. Haas in obtaining permission from Washington DOE to conduct this test are recognized and greatly appreciated.

\section{REFERENCES}

[1] A. Mclnturf, J. Weisend II, C. Dickey, R. Flora, and D. Wallis, "Measured control characteristics of the half cell $\mathbf{4 0} \mathrm{mm}$ aperture magnet sring," Supercollider 4, edited by J. Nonte, Plenum Press, New York , 1992, pp. 859-866.

[2] A. McInmiff, $a$ al., "Half cell 'SSC $40 \mathrm{~mm}$ eperture magnet string," IEEE Trans. on Magn., Vol. 28, p. 512, Jan. 1992.

[3] T. Dombeck, et al., "Cyogenic and power testing of a string of $50 \mathrm{~mm}$ aperture dipole magnets at the Superconducting Super Collider Laboratory," Int. J. Mod. Phys. A, (Proc. Suppl.), 2B, 1993.

[4] A. Mcinturf, et al., "Half cell operational tests of the SSCL," IEEE Trans. on Applied Superconductivity. Vol. 3, pp. 740-743, March 1993.

[5] A. MeInturf, et al., "Test resulss and operational characteristics of prototype SSCL half cell," Proc. of the Thirteenth International Conf. on Magnet Technology, Victoria, B.C., Canada, September 1993.

[6] J. Weisend II, at al., "Heat leak performance of the SSC Collider dipole magnets", Proc. of the Thirteenth International Conf. on Magnet Technology, Victoria, B.C., Canada, September 1993.

[7] W. Robinson, "Locating and clearing the short in the Meyer SPR," SSCL, Collider Group Engineering Note, November 9, 1993, unpublished.

[8] J. Strait, \& al., "Quench performance of Fermilab/General Dynamics built full length SSC Collider dipole magnets," Supercollider 4, edit. J. Nonte, Plenum Press, New York, 1992, pp. 365-372.

[9] P.S. Martin, "Design and operation of the quench protection system for the Fermilab Tevetron," AP Conference Proceedings 184, Physics of Particle Accelerators, Vol. 2, 1989, pp. 2073-2095

[10] J. R. Sanford and D. M. Mathews, Eds., "Site specific concepual design of the Super Conducting Super Collider", SSCL-SR-1056, (1990).

[11] W. Robinson, et al., "Electrical performance characteristics of the SSC ASST," Proc. 1993 Particle Accel. Conf., vol. 4 pp. 2731-2735, IEEE Pub. Cat. No. 93CH3279-7,(1993).

[12] J. Tompkins, "Summaries of Iquench vrs ramp rate studies on full length $50 \mathrm{~mm}$ dipole magnets," SSCL MD TA241, 1993.

[13] A. Devred and T. Ogitsu for SSC Magnet R\&D Collaboration, "Rampmate sensitivity of SSC dipole magnet prototypes," unpublished.

[14] S. Delchamps, et al., "AC loss measurements of SSC dipole magnets," Proc. ICFA Workshop on AC Superconductivity, ACSC92, National Lab for HEP, Tsukula, KEK Proceedings 92-14, 1992.

[15] T. Ogitsu, Y. Zheo, A. Akheiov, and A. Devred, "Influence of cable eddy currents on magnetic field harmonics," Proc. ICFA Workshop on AC Superconductivity, ACSC92, National Lab for HEP, Tsukula, KEK Proceedings 92-14, 1992.

[16] S. Johnson," Maximum temperature in DCA321 magnet coil during spot heater induced quenches", SSCL MDTA-229, 1992. 